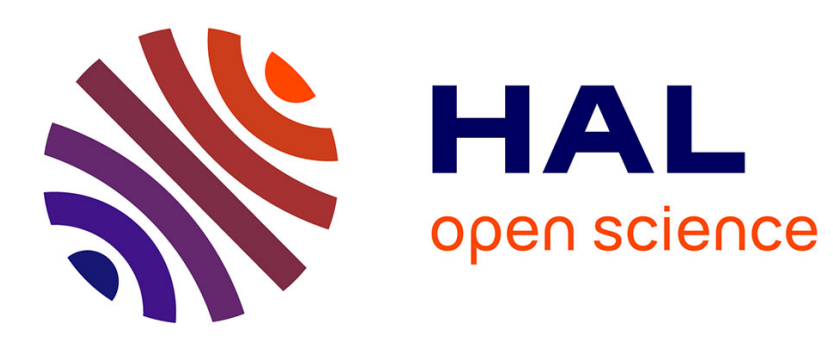

\title{
A Millimeter-Wave Reconfigurable Intelligent Metasurface Design for Vehicular Networks Applications
}

Carola Rizza, Valeria Loscrì, Mohammad Ojaroudi Parchin

\section{To cite this version:}

Carola Rizza, Valeria Loscrì, Mohammad Ojaroudi Parchin. A Millimeter-Wave Reconfigurable Intelligent Metasurface Design for Vehicular Networks Applications. 2020 IEEE Vehicular Technology Conference, Oct 2020, Victoria, Canada. hal-02894947

\section{HAL Id: hal-02894947 https://hal.inria.fr/hal-02894947}

Submitted on 9 Jul 2020

HAL is a multi-disciplinary open access archive for the deposit and dissemination of scientific research documents, whether they are published or not. The documents may come from teaching and research institutions in France or abroad, or from public or private research centers.
L'archive ouverte pluridisciplinaire $\mathbf{H A L}$, est destinée au dépôt et à la diffusion de documents scientifiques de niveau recherche, publiés ou non, émanant des établissements d'enseignement et de recherche français ou étrangers, des laboratoires publics ou privés. 


\section{A Millimeter-Wave Reconfigurable Intelligent Metasurface Design for Vehicular Networks Applications}

\author{
Carola Rizza \\ Inria Lille \\ Lille, 59000 France \\ carola.rizza@inria.fr
}

\author{
Valeria Loscrí \\ Inria Lille \\ Lille, 59000 France \\ valeria.loscri@inria.fr
}

\author{
Mohammad Ojaroudi Parchin \\ Inria Lille \\ Lille, 59000 France \\ mohammad.ojaroudi-parchin@inria.fr
}

\begin{abstract}
A reconfigurable metasurface unit-cell is investigated in order to obtain a $0^{\circ} / 180^{\circ}$ phase shift of the incident millimeter electromagnetic wave and its reflection for automotive applications. The meta-atom is realized starting from a ground and a dielectric layer, over which a metallic pattern is deposited. A diode is added to the pattern in order to make the structure reconfigurable in terms of phase. By tuning the substrate parameters (dielectric thickness $h$ and dielectric constant $\epsilon_{\mathbf{r}}$ ) and with the shape and dimensions of the pattern on the top of it, the unit-cell that satisfies the requirements is found. The $S_{11}$ of the structure is shown both in modulus and phase and compared for the two configurations of the diode. Moreover, a vehicular scenario where a whole reconfigurable intelligent metastructure based on this unit cell is described. The ideal instantaneous rate is also derived.
\end{abstract}

Index Terms - meta-atom, automotive, metasurface, reconfigurability, reflection, phase shift

\section{INTRODUCTION}

Metasurfaces are artificially engineered surfaces, given by the repetition of a unit cell (the meta-atom), that are able to manipulate incident electromagnetic waves in unconventional ways [1]. Changing the polarization and providing phase shift are only some examples. In a wider context, they can be adopted in order to limit the signal deterioration caused by the environment. [2], [3]. Indeed, physical objects significantly alter the propagation of electromagnetic waves producing fading phenomena, due to multi-path propagation, and interference, due to reflections and refractions. Moreover, signals are subjected to attenuation due to absorption losses and to the natural spreading of the power within the space, thus reducing the communication distance [3], [4]. Applying metasurfaces upon all the objects that produce these effects, signal deterioration can be reduced or canceled. Recent literature shows some potential applications of Reconfigurable Intelligent Metasurfaces (RIMs) in wireless networks and how a new era of wireless communication can be paved based on this technology

Signal deterioration becomes really relevant at millimeter waves and at higher frequencies because of molecular absorption, multi-path fading and Doppler effect even at pedestrian speed [4].
So, this issue involves also the automotive area, where the sensors use the $77 \mathrm{GHz}$ band $(76-81 \mathrm{GHz})$. Automotive radar systems are adopted in adaptive cruise control and also in autonomous driving assistance systems where they work for collision avoidance, pedestrian and cyclist detection, and complements vision-based camera-sensing systems [5]. Longrange radars (LRR), that are the automotive sensors that use $77 \mathrm{GHz}$ band, in particular measure the distance from other vehicles and their speed and detect objects in ranges of $80 \mathrm{~m}$ to $200 \mathrm{~m}$ or even greater [6].

Recently, research community has started to focus on millimiter-wave for high bandwidth connected vehicles [7]. The authors show how the most emergent challenge is represented by the increasing amount of data that vehicles produce and need to exchange to each other in order to allow the deployment of new services for safety and critical applications. The main issues in vehicular networks based on millimeterwave are represented by coverage and connectivity [8], [9]. Reconfigurable metasurfaces can be a viable solution to make vehicular networks based on millimeter-wave a fascinating and effective solution thanks to their capabilities of controlling waves and programming the environment. Indeed, the surrounding has been always considered as an obstacle to be defeated and several solutions (e.g. based on cognitive approaches) have tried to overcome the hostility of the environment. In order to accomplish the increasing demand of new services in the vehicular communication context, a new revolutionary paradigm is needed.

In this paper, the structure of a reconfigurable metasurface unit-cell for applications in the automotive sector is presented and its simulated behavior is discussed. By tuning the state of each meta-atom, they can perform beam steering of a signal to a specific direction, thus overcoming constraints due to mobility of the vehicles and the high dynamic in vehicular contexts. The rest of the paper is organized as follows. In Section II we present the specific vehicular scenario considered. In Section III we detail the structure of the proposed meta-atom; In Section IV we show the performance of the proposed structure. In Section V we evaluate the system based on the proposed structure. Finally, we conclude our paper in 
Section VI.

\section{Vehicular COMmunication BASED ON RIS RELAY}

In this section we describe the vehicular network model based on Reconfigurable Intelligent Metasurface (RIM) relays. The relays are represented by the building coated with RIM, whose meta-atom details will be described in the next section.

\section{A. System Description}

Our reference system is represented as a vehicular context where vehicles are deployed on the routes as shown in the Figure 1

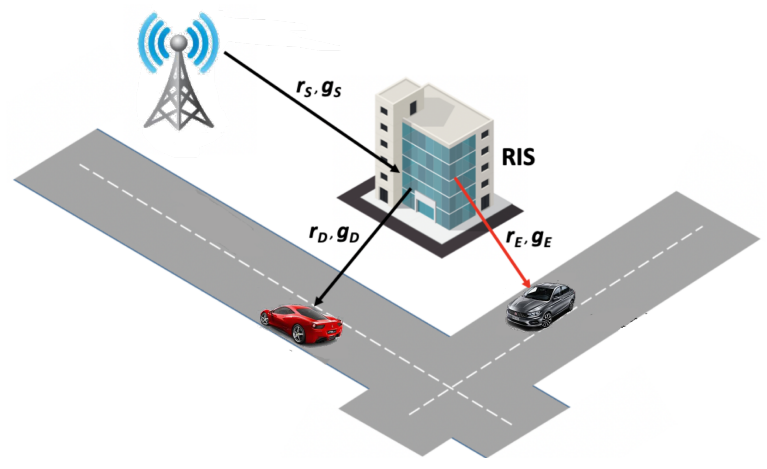

Fig. 1. System model of a vehicular network scenario with RIS (Reconfigurable Intelligent Surface) relays. The whole structure is constituted by $\mathrm{N}$ meta-atom.

In the specific scenario considered in our paper, RIMs are exploited as relays for vehicular nodes. In particular, a building is coated with a reconfigurable intelligent metasurface and it is used as relay from a fixed source (e.g. an RSU, Roadside Unit), while a node D (i.e. the red vehicle in the Figure) is the destination and is a mobile node. There are two wireless links we have to consider. The first one is between the source to the relay, let indicate is as $g_{s}$ and is modeled as a Rayleigh fading channel. The channel between the relay and the destination (i.e. D) is modeled as a doubleRayleigh channel. Our metasurface will be constituted by $\mathrm{N}$ reconfigurable units. Moreover, the RIM is controlled by a software-defined approach for intelligent transmissions. Based on this configuration, the received signal at the destination node can be computed as:

$$
y_{D}=\left[\sum_{n=1}^{N} h_{S, n} h_{D, n} e^{-j \phi_{n}}\right] x+\omega_{D}
$$

where $\mathrm{N}$ is the number of unit cells of the whole metasurface, $x$ is the transmitted signal by the source, $\omega_{D}$ represents Additive Gaussian Noise AWGN at the receiver, $h_{S, n}=$ $\sqrt{g_{S, n} r_{s}^{-\beta} e^{-j \theta_{n}}}$ is the source-to-RIM channel, $r_{s}$ represents the distance, $\theta_{n}$ is the phase of the impinging wave, $g_{s}$ follows a Rayleigh distribution. The term $h_{D, n}=\sqrt{g_{D, n} r_{D}^{-\beta} e^{-j \psi_{n}}}$ represents the channel coefficient from RIM-to-D, the destination node, with a distance $r_{D}$ and a pathloss exponent $\beta, \psi$ is the phase and $g_{D, n}$ follows a double Rayleigh distribution in order to take into account the mobility node [10].

Given that, we can compute the instantaneous SNR at the node $\mathrm{D}$, by implicitly assuming a perfect alignment between the relay and the destination node, as:

$$
\gamma_{D}=\frac{\sum_{n=1}^{N} P_{S}\left|h_{S, n}\right|^{2}\left|h_{D, n}\right|^{2}}{N_{0}}
$$

where $P_{S}$ is the percentage of power of the transmitted signal $x$. Indeed, the whole power associated to the impinging wave on the RIM is $P_{S t o t}$, but a percentage of the whole power will be lost by retransmitting the signal towards the destination node. In order to maximize the SNR at destination, we need to keep this percentage as small as possible. The design of the meta-atom presented in the next section is realized in such a way to minimize this power loss component and to work at the millimeter wave frequency.

\section{THE DESIGN OF OUR META-ATOM}

In this section, a particular meta-atom structure with reflective capabilities working in the range $76-81 \mathrm{GHz}$ is presented. It consists of a metallic pattern with a four-leaf clover shape, over a dielectric substrate and a ground plane, as shown in Fig. 2. In the metallic pattern, a diode connected to a via hole is added in order to make the structure reconfigurable in terms of phase. The via hole connects the metallic pattern upon the substrate to the ground plane.

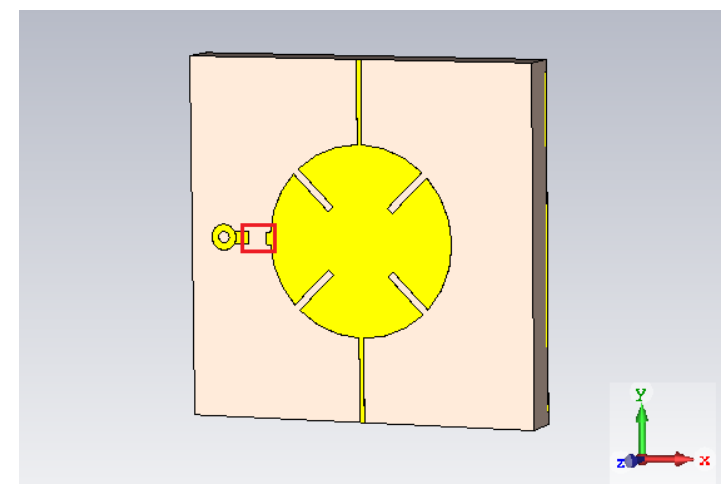

Fig. 2. Meta-atom structure consisting of a metallic pattern, a dielectric substrate (Rogers RT5870) and a ground plane. The highlighted section represents a diode in "off" state.

The substrate is made of Rogers RT5870, whose dielectric constant is 2.33 and the electric tangent loss is 0.0012. It has a square shape with each side of length $2.679 \mathrm{~mm}$ (that corresponds to $\lambda_{0} / 1.426 \mathrm{~mm}$ where $\lambda_{0}$ is the free space wavelength at the central frequency of $\mathrm{f}_{0}=78.5 \mathrm{GHz}$ ) and its thickness is $0.321 \mathrm{~mm}\left(\lambda_{0} / 11.9 \mathrm{~mm}\right)$. The pattern upon the substrate is made of copper and it has been designed starting from a circumference with radius $0.714 \mathrm{~mm}\left(\lambda_{0} / 5.35 \mathrm{~mm}\right)$, where 4 slots have been done of width $0.054 \mathrm{~mm}$ and length $0.383 \mathrm{~mm}$. The via hole has an external radius of $0.098 \mathrm{~mm}$ and an inner radius of $0.043 \mathrm{~mm}$. The via hole is connected to 
the slotted circumference through a diode, that for simplicity has been modeled with an open circuit when it is in "off" state and a short circuit when it is in "on" state. The open circuit has a length of $0.141 \mathrm{~mm}$ and a width of $0.089 \mathrm{~mm}$. In the $y$ direction two biasing lines have been added in preparation for the entire metasurface made of the repetition of many identical unit-cells, where only the state of the diode can be modified. The aforementioned structure has been simulated on CST (Computer Simulation Technology) [11]. In order to do simulations, it was necessary to establish the area of computation setting the Boundary Conditions. According to the chosen settings, a larger structure consisting of more meta-atoms (a sort of small metasurface) has been simulated. These settings have established also the illumination of the structure. Floquet ports have been created that allow to generate plane waves. The number of excited modes has been set to two, that correspond to the two fundamental modes, $\operatorname{TE}(0,0)$ and $\operatorname{TM}(0,0)$, that are linearly polarized plane waves. However, the results shown in the section IV refer only to $\operatorname{TM}(0,0)$ mode.

\section{EVALUATION OF THE META-ATOM}

First of all, it is proved that the previously-described structure behaves like a metasurface unit-cell. Thus, the effective permittivity $\epsilon_{\text {eff }}$ and permeability $\mu_{\text {eff }}$ of the meta-atom have been computed on Matlab using formulas (3) - (7) [12].

$$
\begin{aligned}
& \mathbf{z}=\sqrt{\frac{\left(\mathbf{1}+\mathbf{S}_{11}\right)^{2}-\mathbf{S}_{21}{ }^{2}}{\left(\mathbf{1}-\mathbf{S}_{11}\right)^{2}-\mathbf{S}_{21}{ }^{2}}} \\
& \mathbf{n}=\frac{\mathbf{1}}{\mathbf{k}_{0} \mathbf{d}}\{[\mathbf{I m}[\ln (\psi)+\mathbf{2} \mathbf{m} \pi]-\mathbf{i R e} \ln (\psi)]\} \\
& \psi=\frac{\mathbf{S}_{21}}{\mathbf{1}-\mathbf{S}_{21 \frac{\mathbf{z}-\mathbf{1}}{\mathbf{z}+\mathbf{1}}}} \\
& \epsilon_{\text {eff }}=\frac{\mathbf{n}}{\mathbf{z}} \\
& \mu_{\text {eff }}=\mathbf{n z}
\end{aligned}
$$

The obtained graphs are shown for both the configurations in Figs. 3 and 4. For limits of space, only the real parts of the effective permittivity and permeability are shown here. It is worth noticing that, around the central frequency 78.5 $\mathrm{GHz}$, in the on-state meta-atom the effective permittivity is positive while the effective permeability is negative. Looking at the other configuration, this behaviour is reversed, since the permittivity is almost zero but negative and the permeability is positive. Since in both cases at least one parameter between $\epsilon_{\text {eff }}$ and $\mu_{\text {eff }}$ has negative values, the structures behave like metasurface unit-cells.

Then, the scattering parameters have been computed for both the states of the diode. In particular, the reflection coefficient $S_{11}$ and the transmission one $S_{21}$ have been taken in greater account. Indeed, in order to build metasurfaces to be applied in the automotive domain, it is required that the unit-cell maximize the reflected signal. This is translated in having a reflection coefficient almost equal to $0 \mathrm{~dB}$ in the frequency

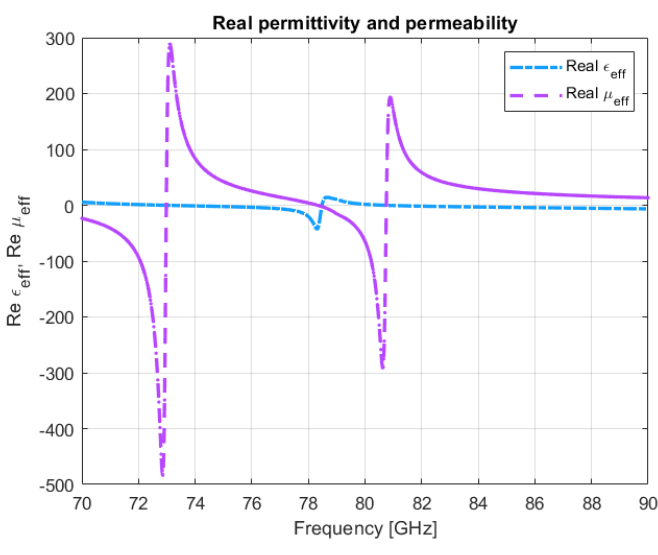

Fig. 3. Real part of the effective permittivity and permeability in case of the structure of Fig. 2 with diode in on state.

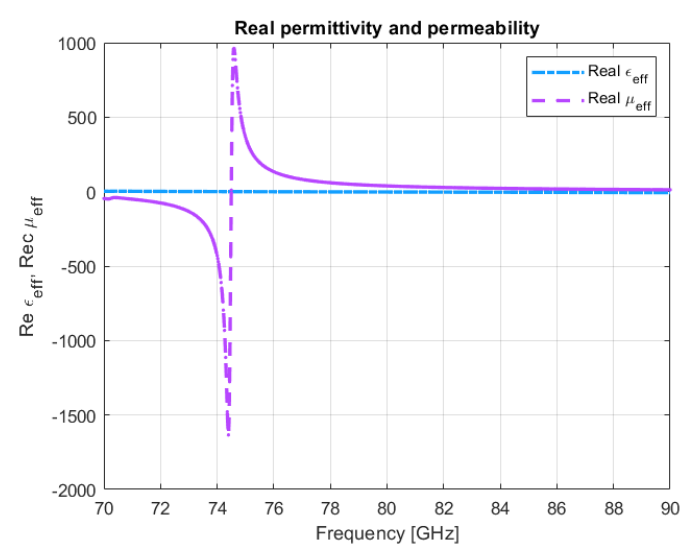

Fig. 4. Real part of the effective permittivity and permeability in case of the structure of Fig. 2 with diode in off state.

range of interest and, simultaneously, in maintaining the transmission coefficient much lower than the reflection one (at least $30 \mathrm{~dB}$ lower). The obtained reflection and transmission coefficients, in modulus, are shown in Figs. 5 and 6.

For both the states of the meta-atom, the $S_{11}$ is very closed to $0 \mathrm{~dB}$ : the lowest value is $-3.82 \mathrm{~dB}$ and happens for the case with the diode in on state. As regards the transmission coefficient $\mathrm{S}_{21}$, it is, in both cases, lower than $-40 \mathrm{~dB}$, so the amount of transmitted signal is negligible.

In order to obtain a metasurface which can steer the direction of the reflected signal in a wide angular range, it is required that the phase of the signal reflected by each unit-cell can be switched between $0^{\circ}$ and $180^{\circ}$. In order to reach this aim, the phase of the reflection coefficient is required to have a shift of $180^{\circ}$ when the state of the diode is changed. The phase of the two reflection coefficients ( for "on" state and for "off" state) is shown in Fig. 7, while Fig. 8 shows the difference among the two curves. It is worth noticing that the higher difference between the two phase curves happens around $78 \mathrm{GHz}$. More precisely, the phase difference reaches $180^{\circ}$ in $78.52 \mathrm{GHz}$. So, the phase change happens at the central frequency of the band 


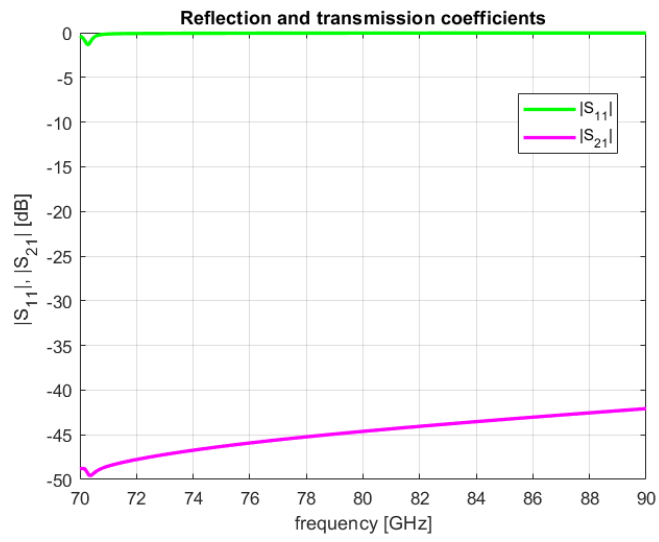

Fig. 5. Reflection coefficient $S_{11}$ and transmission coefficient $S_{21}$ in modulus of the meta-atom of Fig. 2 with diode in off state.

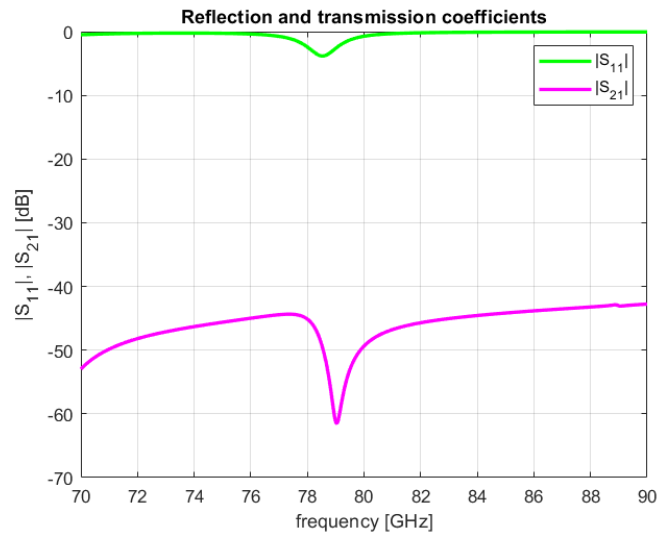

Fig. 6. Reflection coefficient $S_{11}$ and transmission coefficient $S_{21}$ in modulus of the meta-atom of Fig. 2 with diode in on state.

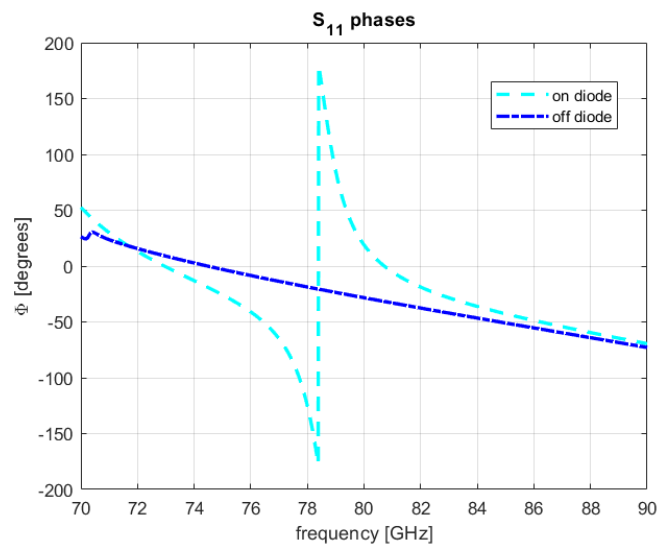

Fig. 7. Phase curve of the $S_{11}$ for the two states of the diode.

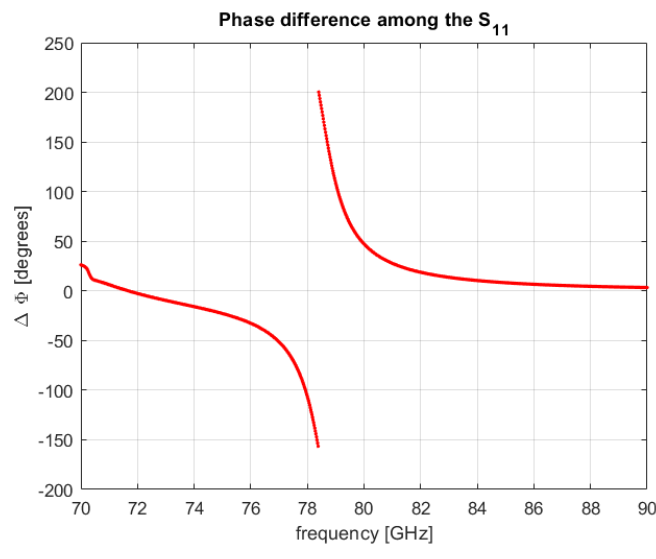

Fig. 8. Phase difference between the $\mathrm{S}_{11}$ in "off" and "on" state of the diode.

adopted by automotive sensors.

\section{REsults And Discussion}

In this Section, we consider a structure composed by $N \times N$ meta-atom described in the previous sections and we evaluate the impact of changing the transmitting power $P_{s}$.

In particular, based on the equation (2) of the instantaneous $\mathrm{SNR}$, we evaluate the received power as:

$$
P_{r x}\left(t, \phi_{b}\right)=k\left(\frac{\pi^{2}}{\phi_{b}^{2}}\right)^{2} \frac{1}{\left[(v t)^{2}+(d)^{2}\right]^{\beta / 2}}
$$

where $\phi_{b}$ accounts the phase component by considering different beamwidth of the wave, $d$ is the distance between the relay and the receiver vehicle and $k$ is defined as $10 \log _{10}(k)=$ $P_{s}(d B m)-E+10 n \log _{10}(\lambda / 4), E=10 d B$ is the shadowing margin and $\lambda$ is the carrier wavelength.

$$
P_{\text {noise }}=-174+10 * \log _{10} B+N F
$$

where B is the bandwidth of the system and NF is a noise figure to account the velocity in the system [13].

We consider an ideal beam model where the gain inside the beam is uniform and zero outside. The instantaneous SNR can be rewritten as:

$$
\left.\operatorname{SNR}\left(t, \theta_{b}\right)\right)=P_{r x\left(t, \theta_{b}\right)} / P_{\text {noise }}
$$

The instantaneous rate can be derived as:

$$
R(t)=\log 2\left(1+\lambda_{D}(t)\right)
$$

The parameters used in this evaluation are summarized in Table I

In Figure 9 and 10 we evaluate the ideal instantaneous rate in respect of different power of the transmitter. It is worth to notice that we evaluated the transmitter power till a maximum value of $80 \mathrm{~dB}$ but the EIRP (Effective, or Equivalent, Isotropically Radiated Power), namely the maximum amount of power that could be radiated from an antenna, given its 
TABLE I

SiMULATION PARAMETERS

\begin{tabular}{|c|c|}
\hline Parameter & Value \\
\hline$P_{s}$ & {$[0,20,40,60,80] \mathrm{dBm}$} \\
\hline $\mathrm{B}$ & $2.16 \mathrm{GHz}$ \\
\hline$f_{c}$ & $6 \mathrm{GHz}$ \\
\hline $\mathrm{d}$ & $5,60,120 \mathrm{~m}$ \\
\hline $\mathrm{NF}$ & $6 \mathrm{~dB}$ \\
\hline$v$ & $80 \mathrm{Km} / \mathrm{h}$ \\
\hline$\beta$ & 2.7 \\
\hline$\phi_{b}$ & $30^{\circ}, 120^{\circ}$ \\
\hline
\end{tabular}

antenna gain and the transmitter power of the RF system in Europe is $57 \mathrm{~dB}$.

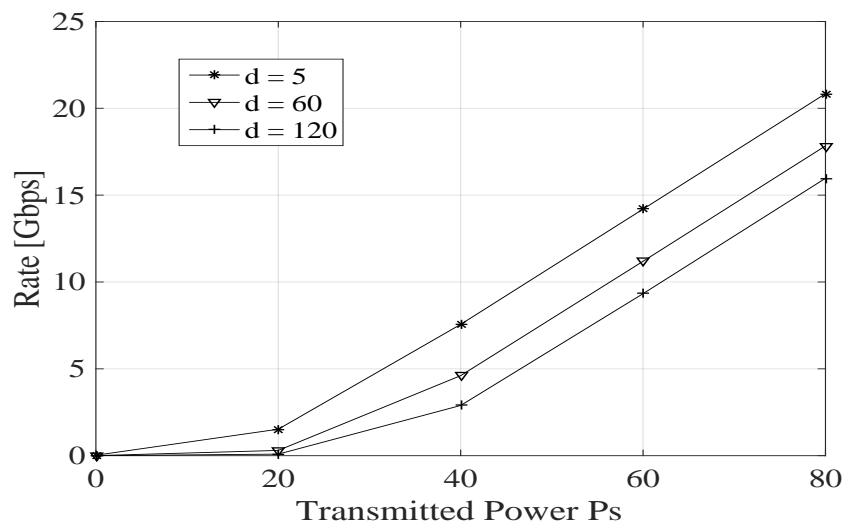

Fig. 9. Evaluation of the instantaneous rate vs the transmitter power with a beamwidth $\phi_{b}=30^{\circ}$

Of course, at higher distance, the instantaneous rate decreases. The maximum value of instantaneous rate is achieved for a distance of 5 meters with a beamwidth equal to $30^{\circ}$ and is $\simeq 21 \mathrm{Gbps}$. In the same conditions of distance but with a beamwidth equal to $120^{\circ}$ we have $\simeq 17 \mathrm{Gbps}$. It is worth to recall that we are considering the value represented in the Figures 9 and 10 as a kind of benchmark by assuming that the whole structure constituted by the meta-atom proposed can realize that the whole gain is in the main beam and zero outside. Moreover, we are considering a system with a perfect beam tracking, where the SNR is always maximized.

\section{CONCLUSION}

Vehicular communication is a paradigm characterized with a very high dynamic and variable scenario. Communication can be between two vehicles or a fixed node (RSU) and a vehicle. In all the cases, the velocity of the nodes impact in a very important way on the performance of the communication protocols. A new paradigm based on the exploitation of Reconfigurable Intelligent Metasurface (RIM), could play an important role for pushing a novel category of communication protocols where the environment is reprogrammed and be part of the communication protocol design.In this paper we have investigated and proposed a specific meta-atom characterized with specific features in terms of reconfigurable phase at

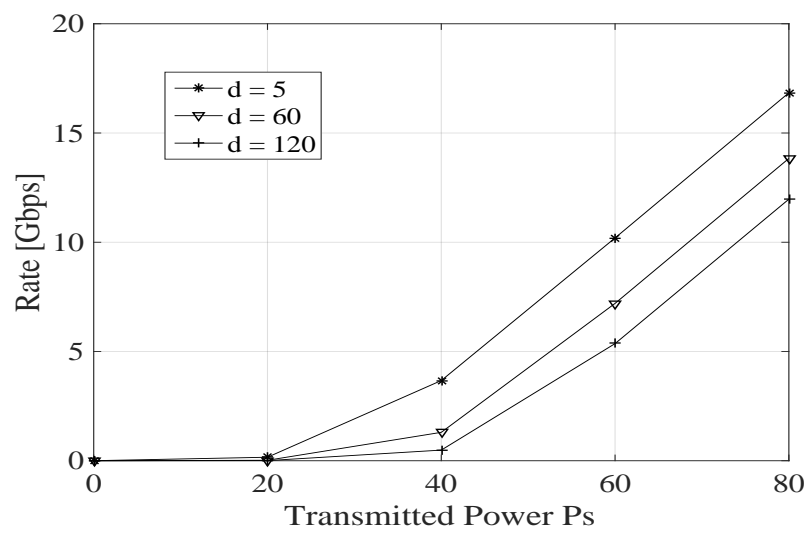

Fig. 10. Evaluation of the instantaneous rate vs the transmitter power with a beamwidth $\phi_{b}=120^{\circ}$.

millimeter frequency. This unit cell is the basic component for a whole reprogrammable metasurface. By characterizing the vehicular channel, we have derived the ideal instantaneous rate. In future work, we will implement and test the whole metasurface and will compare the performance in a vehicular scenario by comparing its behavior with the ideal one obtained.

\section{REFERENCES}

[1] L. Zhang, X. Q. Chen, S. Liu, Q. Zhang, J. Zhao, J. Y. Dai, G. D. Bai, X. Wan, Q. Cheng, G. Castaldi, V. Galdi, and T. J. Cui, "Space-TimeCoding Digital Metasurfaces," 2019 Thirteenth International Congress on Artificial Materials for Novel Wave Phenomena (Metamaterials), Rome, Italy, 2019, pp. X-128-X-130.

[2] Marco Di Renzo, Jian Song. Reflection probability in wireless networks with metasurface-coated environmental objects: an approach based on random spatial processes. EURASIP Journal on Wireless Communications and Networking, SpringerOpen, 2019.

[3] E. Basar, M. Di Renzo, J. De Rosny, M. Debbah, M. Alouini and R. Zhang, "Wireless Communications Through Reconfigurable Intelligent Surfaces," in IEEE Access, vol. 7, pp. 116753-116773, 2019.

[4] Christos Liaskos, Shuai Nie, Ageliki Tsioliaridou, Andreas Pitsillides, Sotiris Ioannidis, and Ian Akyildiz,"A New Wireless Communication Paradigm through Software-Controlled Metasurfaces," IEEE, 2018.

[5] M. Parker,"Digital Signal Processing 101 (2 ${ }^{\text {nd }}$ Edition)," pp. 253-276, 2017.

[6] https://www.everythingrf.com/community/automotive-radar-basics, accessed on $27^{\text {th }}$ April 2020.

[7] J. Choi, V. Va, N. Gonzalez-Prelcic, R. Daniels, C. R. Bhat and R. W. Heath, "Millimeter-Wave Vehicular Communication to Support Massive Automotive Sensing," in IEEE Communications Magazine, vol. 54, no. 12, pp. 160-167, December 2016.

[8] Marco Giordani, Mattia Rebato, Andrea Zanella, Michele Zorzi, Coverage and connectivity analysis of millimeter wave vehicular networks, Ad Hoc Networks, Volume 80, 2018, Pages 158-171.

[9] Giordani, Marco et al. "Millimeter wave communication in vehicular networks: Challenges and opportunities." 2017 6th International Conference on Modern Circuits and Systems Technologies (MOCAST), 1-6, (2017).

[10] Y. Ai, M. Cheffena, A. Mathur, and H. Lei, "On Physical Layer Security of Double Rayleigh Fading Channels for Vehicular Communications," IEEE Wireless Commun. Lett., vol. 7, no. 6, pp. 1038-1041, Dec. 2018.

[11] Microwave Studio, "Computer Simulation Technology," 2017.

[12] Josep Canet-Ferrer,"Metamaterials and metasurfaces," Canet-Ferrer, Josep, (editor.) and IntechOpen (Firm), (issuing body.) Metamaterials and metasurfaces. IntechOpen, London, United Kingdom, 2019.

[13] J. Kim and A. F. Molisch, "Enabling Gigabit services for IEEE 802.11ad-capable high-speed train networks," in IEEE Radio and Wireless Symposium (RWS), pp. 145-147, Jan. 2013. 